\title{
An Adaptive Approach Digital Image Watermarking based on Discrete Wavelet Transform (DWT)
}

\author{
Pramod Kumar ${ }^{1}$, Prakarti Triwedi ${ }^{2}$, Surbhi Sharma ${ }^{3}$ \\ ${ }^{I}$ Computer Science \& Engineering, Govt. Engineering College, Ajmer, India \\ ${ }^{2}$ Assistant Professor, Computer Science \& Engineering, Govt. Engineering College, Ajmer, India \\ ${ }^{3}$ Assistant Professor, Computer Science \& Engineering, Aryabhatta \\ College of Engg. \& Research Center, Ajmer, India \\ Igpupn51@gmail.com, 2niyuvidu@rediffmail.com, ${ }^{3}$ surbhisharma26@gmail.com
}

\begin{abstract}
The applications of different dimension of multimedia have been grown rapidly on daily basis. Digital media brings about the changes in the conveniences to the people, The cons of this technology is security threat if that security issue exist there is no meaning of conveniences We have segmented the proposed work in such a way unlike conventional approach the module of work includes sub-plotting the image in three directional coordinates $\operatorname{plot}(x)$, plot $(g), \operatorname{plot}(y)$. The security of information may have the distinct dimension in growing effective techniques to discourage the unauthenticated technique of duplication of virtual signals. Digital watermarking is the mathematical technique of embedding information right into a virtual signal in a way that it is difficult to eliminate. in order to overcome this, robust dwt watermarking approach is proposed. We've contemplated a way a good way to use $t$ the temporary statistics to apply the inversion of dwt in row way and decompose the picture in the same length of width and height. Robustness can be defined as a watermark to stay unaffected even if digital content is passed thru diverse approaches and attacks. we've got proposed invisible sturdy watermarking for you to proved to be is the most accurate method. in conjunction with conventional technique embed a watermark containing key facts consisting of authentication codes.

We have considered different dynamic conditions where the copyright and basic constituent of distributed images/photos is violated. Handiest one picture in active mode and all different is inactive mode. Since the images/pics posted on social networks or any networks are normally changed and/or compressed to the original template supplied through the carrier carriers, we suggest a digital photo watermarking based on dwt coefficients modification for use for such snapshots/images. we've got carry out the watermarking with proposed approach for the bilateral method which means for encrypting the digital information and for retrieval of the original information from the encoded dataset have alerted the convention concept of Dwt by adding an Adaptive filter into it for extracting the data.The contemplated approach has reduced the noise and unnecessary constituent to provide better efficiency and retrieve the accurate original image without distorting the pixel vectors.
\end{abstract}

IndexTerms-MATLAB, rsa, DWT, watermarking

I. INTRODUCTION

$\mathrm{V}$ IRTUAL watermarking describes techniques and generation that hide facts, for instance a spread of or text, in virtual media, on the facet of pix, video or audio. The embedding takes vicinity via manipulating the content material fabric cloth material of the virtual information, because of this that the statistics is not embedded within the frame at some stage within the facts. The hiding manner wants to be such that the changes of the media are imperceptible. For pix, this means that that the modifications of the pixel values want to be invisible. Furthermore, the watermark wants to be each robust or fragile, relying on the software. Through "robust", we advise the functionality of the watermark to stand as hundreds as manipulations of the media, which embody lossy compression (wherein compressing facts and then decompressing it retrieves records that can be particular from the right, however is near sufficient to be beneficial in some way), scaling, and cropping, amongst others. In some times, the watermark can also want to be fragile. "fragile" technique that the watermark need to now not face up to tampering, or need to face as much as splendid as a good deal as a superb, predetermined quantity.

The instance under illustrates how virtual watermarking can cover statistics in a very invisible way. The proper image is at the left; the watermarked photograph is on the right and consists of the selection of the author.

Wwatermarking is defined as such as (embedding) a watermark signal to. A widespread scheme for virtual watermarking is given in determines 1.1. The watermark message can be a emblem photo, occasionally a visually recognizable binary image or it can be binary bit waft. A watermark is embedded to the host data with the aid of way of the usage of a secret key on the embedded.

\section{SORTS OF TASK SCHEDULING ALGORITHM}

Digital watermarking is a prominent discipline of research and lots of researchers have advised a big quantity of algorithms and as compared. the main thrust on all such algorithms is to hide secrete information (watermark) in host signal in such a way that it offers appropriate tradeoff among imperceptibility and robustness against distinct assaults. this segment gives several kinds of virtual watermarking techniques found in the instructional literature. we do now not 


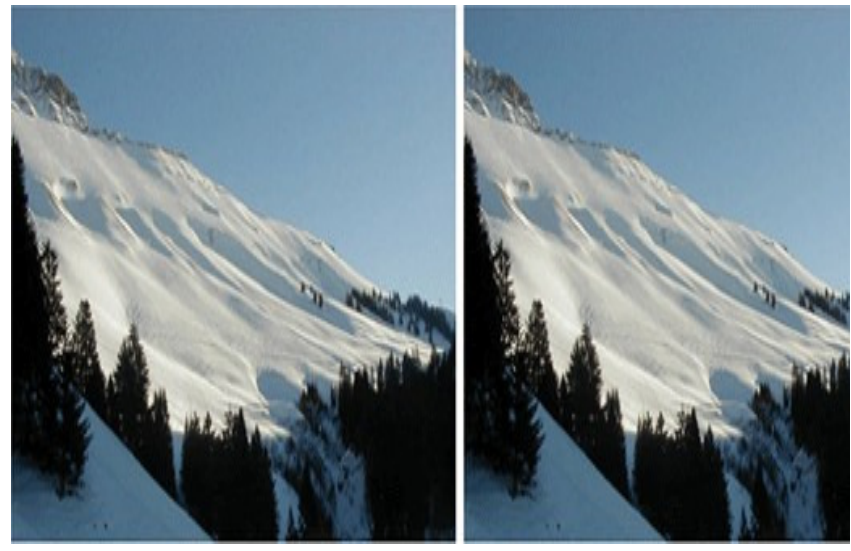

Fig 1.1: A Digital Watermarking System

deliver an exhaustive assessment of the area, however offer an outline of installed approaches. current virtual watermarking techniques are broadly categorized into classes relying at the area of watermark insertion: spatial area and frequency domain techniques.

In comparison to the spatial-domain-based totally definitely watermarking, frequency-region based totally strategies can embed more bits of watermark and are more sturdy to assault; consequently, they're extra attractive than the spatial-vicinity-primarily based strategies, due to the fact the watermark information can be spread out to the complete photo. As to the frequency transform, there are dft (discrete fourier rework), dct (discrete cosine remodel), and dwt (discrete wavelet redesign).

The sooner watermarking techniques are nearly spatial primarily based completely method. In spatial place the watermark is embedded into the host image by way of manner of using the usage of the usage of without delay improving the pixel values, i.e. First-rate example is to embed the watermark inside the least considerable bits (lsbs) of photo pixels [1]. Spatial area watermarking is simple to position into impact and requires no unique photograph for watermark detection. But, it frequently fails underneath signal processing assaults which consist of filtering and compression and having relative low-bit functionality. A easy photograph cropping operation may also moreover additionally eliminate the watermark. Besides, the constancy of the particular image facts may be significantly degraded because of the truth the watermark is at once finished on the pixel values.

\section{Problem Formulation and Proposed Solution}

In this work we implement ECC Algorithm with altered approach for encrypting and decrypting the probe image and encrypted images respectively. Our proposed approach has proposed that the elapsed time to perform the task for same input image is less than the conventional approach. We have run the cryptography procedure for $n$ number of iterations in order to get the accurate results and compared that particular elapsed time with the conventional approach. We have contemplated an approach in which we consider region of interest (ROI) unlike the conventional approach, the advantage of the proposed work is we $\mathrm{n}$ need not to cover all the pixel vectors but only those which is required to be encrypted or decrypted. The proposed work has applied ecc algorithm for error calculations which has been shown in the experimental results.

Three.1 comparison of proposed algorithm with present paintings

Inside the current method, we use the watermarking approach in whole image on the same time. That is the hard manner to apply this method and show the watermarking photograph. There has been many proposed novel strategies to hide watermark in digital photos. Those strategies can be classified into awesome classes consistent with several requirements. The number one criterion is the shape of area in which the facts embedding takes vicinity. There are two maximum critical region types, spatial and remodel domains. The redesign domain picture is represented in terms of its frequencies; however, in spatial place it's miles represented by way of the use of manner of pixels. The second one criterion is in step with the ability of watermark to face up to assault;

In the proposed work, we use the dwt technique to watermarking the photo. On this technique, we've decompose the picture in row and column manner and study one at a time row and column respectively. This the correct way to apply the dwt approach in watermarking picture.

Step 1. First we have considered image and determine the pixel value.

Step 2. Then we use the wfilter(wavelet filter) to filter the image.

Step 3. We decompose the image for clear visibility.

(a) First we have decomposed the image in row manner.

(b) Then we have decomposed the image in column manner.

Step 4. We have use to temporary data to apply the inversion of dwt in row manner

Step 5. Decompose the image in equal size of width and height.

Step 6. Only one image in active mode and all other is not active mode.

Step 7. PSNR is peak signal to noise ratio which is used to define the accuracy of image.

Step 8. In last step we get the dwt watermarking final (single) image.

\section{Result}

\section{A. Matlab Results}

We use the domain based (db) filter having length 6 then read the image(Barbara.png) and show the Barbara image by using imshow command. In this fig we use the wfilter(wavelet filter) to filter the image. A wavelet is a wave-like oscillation with amplitude that begins at zero, increases, and then decreases back to zero. 


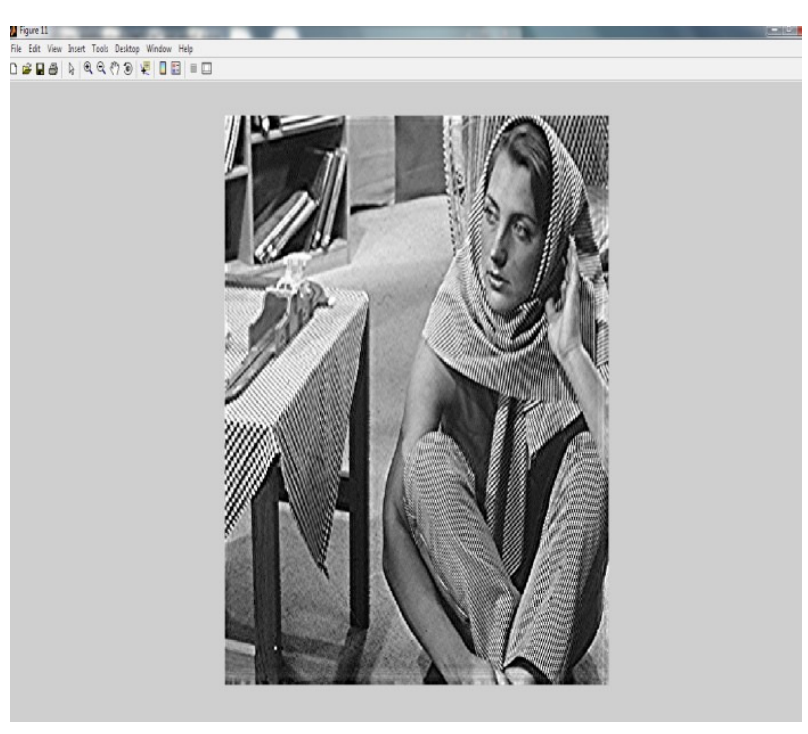

Fig 5.1: wavelet filter

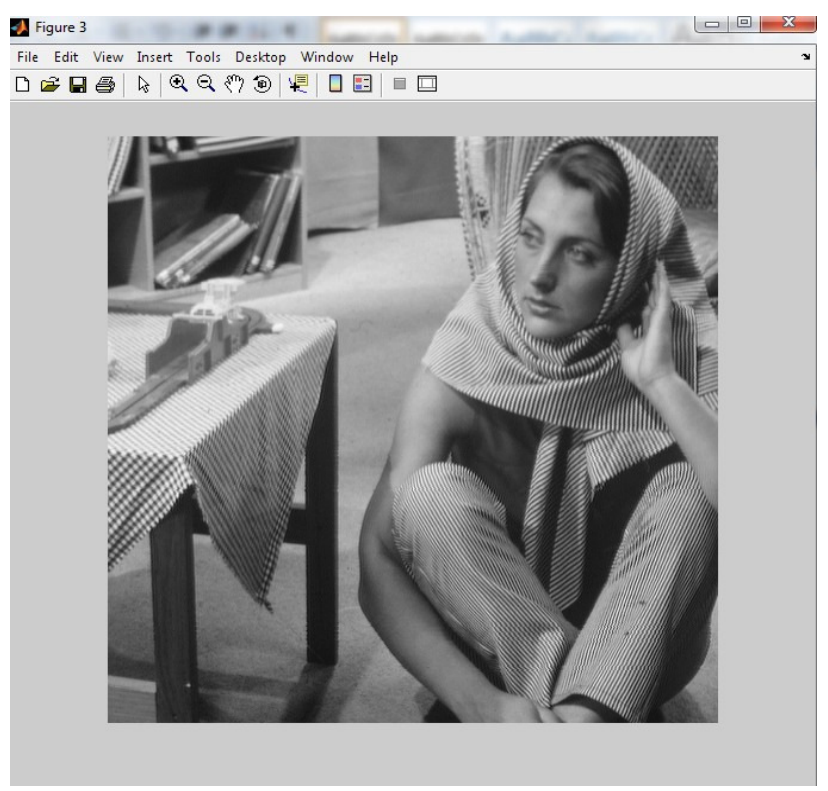

Fig 5.2: convert the 2D image in dwt and idwt

In this fig. we have sub-plotting the image in three direction coordinates $\operatorname{plot}(\mathrm{x}), \operatorname{plot}(\mathrm{g}), \operatorname{plot}(\mathrm{y})$. After that convert the $2 \mathrm{D}$ image in dwt and idwt. By using plotting we enhance the image quality. By using the plotting, we decompose the image for clear visibility. In this fig, we have to mapping the image in row manner that means we have to read the image in row by row.

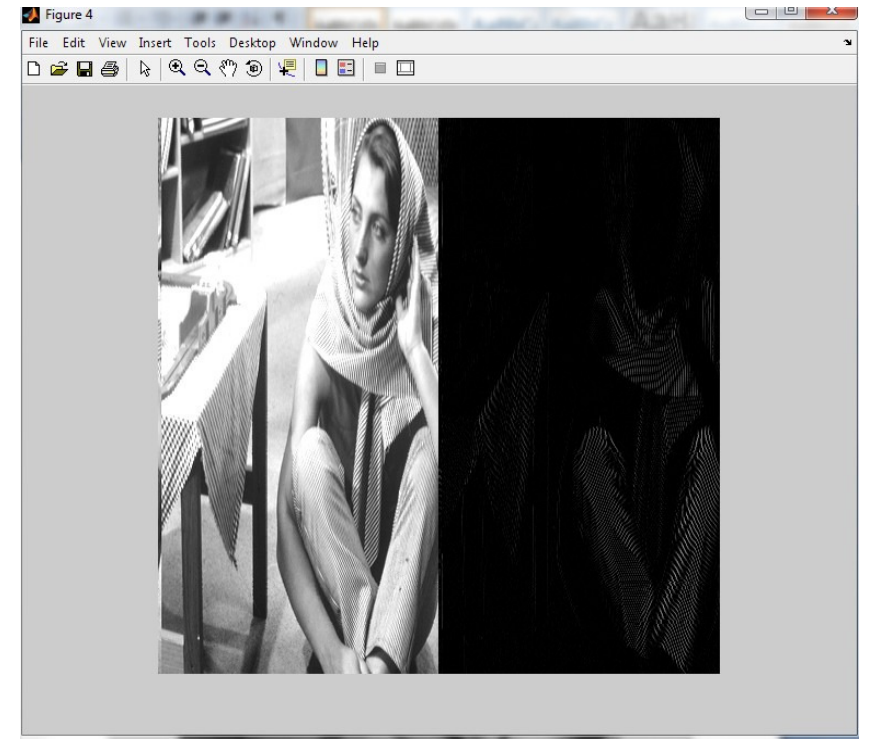

Fig 5.3: mapping the image in column manner

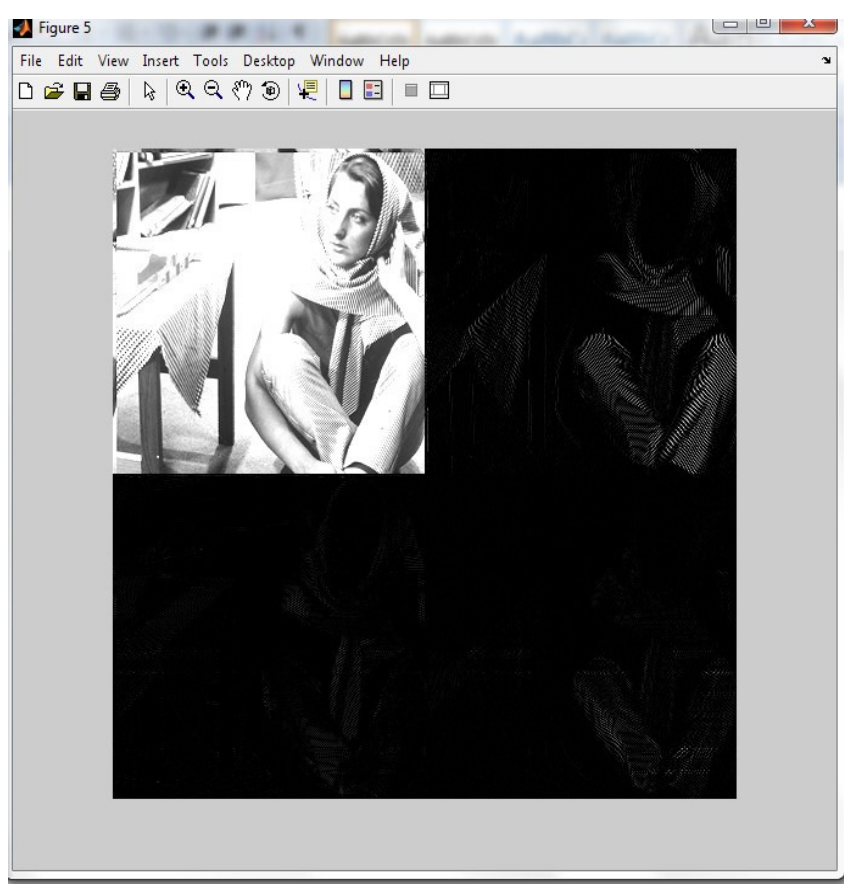

Fig 5.4: decompose the image in equal size of width and height

In this fig. we have use to temporary data to apply the inversion of dwt in row manner and decompose the image in equal size of width and height 


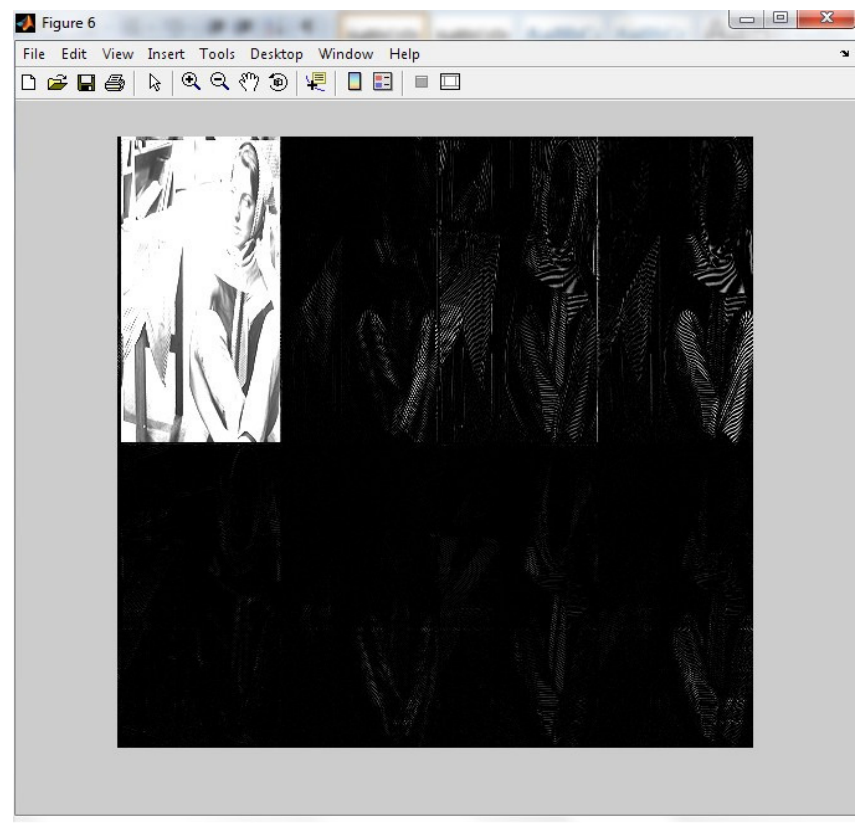

Fig 5.5: inversion of dwt in column manner

In this fig. we have use to temporary data to apply the inversion of dwt in column manner because of temporary data we can handle the image in secure and easy way. All the module of image are read in column way.

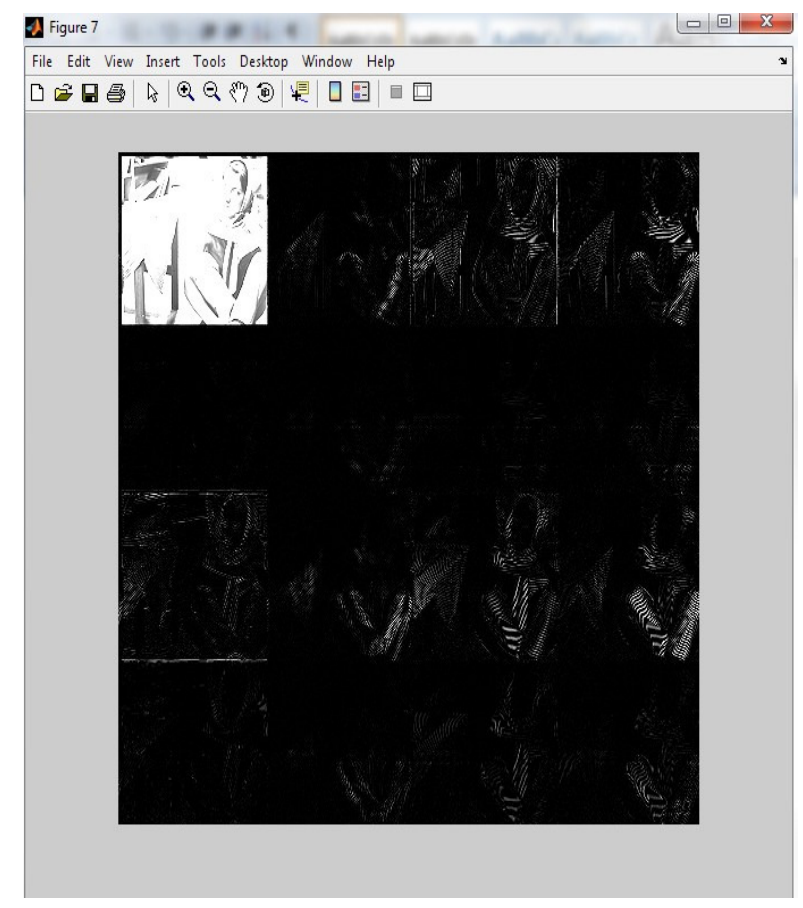

Fig 5.6: one image in active mode and all other is not active mode
In this fig. we have decompose the image in row and column manner simantenously. Only one image in active mode and all other is not active mode. We have filtered the image and apply the watermarking technique at this time.

\section{Conclusion and Future Work}

Interior our research, we've characterized and tested giant photograph segmentation calculations. Mage segmented calculations have a promising future earlier thinking about they'll be the concept of photograph getting prepared and laptop imaginative and prescient and feature have become the center of present day research. But quite a few years of studies, there may be no commonly said algorithm segmentation calculation. Due to the fact that picture segmentation is motivated by manner of bunches of components, for instance, kind of picture, shading, threshold, stage of noise, etc. Alongside those lines there's no single calculation that is pertinent on a large form of photos and nature of problem. Due to every single above element, photo segmentation nevertheless stays a number one pending hassle inside the ranges of picture making ready.

In present technique we had labored on a static photo, in destiny the paintings may be executed on a shifting photo (video).

\section{REFERENCES}

[1] Van Schyndel, R. G., Tirkel, A. Z., and Osborne, C. F., "A digital Watermark." Proc. of the IEEE Int. Conference on Image Processing. Vol. 2, (1994): pp. 86-90.

[2] Swanson, M. D., Kobayashi, M., and Tewfik, A. H., "Multimedia Data-Embedding and Watermarking Technologies." Proc. of the IEEE. Vol. 86, No. 6, (June 1998): pp. 1064- 1087.

[3] Petitcolas, F., Anderson, R., and Kuhn, M., "Information Hiding - a Survey." Proc. of the IEEE. Vol. 87, No. 7, (July 2016): pp. 10621078.

[4] Barni, M., Bartolini, F., Cox, I. J., Hernandez, J., and Perez-Gonzalez, F., "Digital Watermarking for Copyright Protection: A communications perspective." IEEE Communications Magazine. Vol. 39, No. 8, (August 2015):pp. 90-133.

[5] Langelaar, Gerhard C., Setyawan, I., and Lagendijk, R. L., "Watermarking Digital Image and Video Data: A state-of-the-artoverview." IEEE Signal Processing Magazine. Vol. 17, No. 5, (September 2015): pp. 20-47.

[6] Voyatzis, G., Mikolaides, N., and Pitas, I., "Digital watermarking: An overview." Proc. of IX European Signal Processing Conference (EUSIPCO), Island of Rhodes, Greece. (September 8-11, 2014): pp. 13-16.

[7] Wolfgang, R. B., Podilchuk, C.I ., and Edward J. Delp, "Perceptual Watermarks for Image and Video." Proc. of the IEEE. Vol. 87, No. 7, (July 2013): pp. 1109-1126.

[8] Cox, I. J., Miller, M. L., and Bloom, J. A., "Watermarking Applications and their Properties." Proc. of IEEE Int. Conference on Information Technology, Las Vegas. (March 2012): pp. 6-10.

[9] Craver, S., Memon, N., Yeo, B.-L., and Yeung, M. M., "Resolving Rightful Ownerships with Invisible Watermarking Techniques: Limitations, Attacks and Implications." IEEE Journal On Selected Areas in Communications. Vol. 16, No. 4, (May 1998): pp. 573-586.

[10] Voloshynovskiy S. et al., "Attacks on Digital Watermarks: Classification, Estimation- Based Attacks, and Benchmarks." IEEE Communication Magazine. Vol. 39. No. 8, (August 2012): pp. 118-126. 